\title{
Dispersible Colloid Facilitated Release of Organic Carbon From Two Contrasting Riparian Sediments
}

\author{
Kenton A. Rod ${ }^{1 * t}$, Kaizad F. Patel ${ }^{1}$, Swatantar Kumar ${ }^{1}$, Elizabeth Cantando ${ }^{2}$, \\ Weinan Leng ${ }^{2}$, Ravi K. Kukkadapu ${ }^{3}$, Odeta Qafoku ${ }^{3}$, Mark Bowden ${ }^{3}$, Daniel I. Kaplan ${ }^{4}$ and \\ Kenneth M. Kemner ${ }^{5}$ \\ ${ }^{1}$ Pacific Northwest National Laboratory, Richland, WA, United States, ${ }^{2}$ Virginia Tech National Center for Earth and \\ Environmental Nanotechnology (NanoEarth), Blacksburg, VA, United States, ${ }^{3}$ Environmental and Molecular Sciences \\ Laboratory, PNNL, Rlchland, WA, United States, ${ }^{4}$ Savannah River National Laboratory, Aiken, SC, United States, \\ ${ }^{5}$ Bioscience Division, Argonne National Laboratory, Lemont, IL, United States
}

OPEN ACCESS

Edited by:

Carl I. Steefel,

Lawrence Berkeley National Laboratory, United States

Reviewed by:

Wenming Dong,

Lawrence Berkeley National Laboratory, United States Mohammad Zafar Afsar, University of Delaware, United States

*Correspondence: Kenton A. Rod kenton.rod@gmail.com

tPresent address: Kenton A. Rod

Washington State Department of Ecology, Richland, WA, United States

Specialty section:

This article was submitted to

Water and Critical Zone,

a section of the journal

Frontiers in Water

Received: 10 May 2020 Accepted: 30 November 2020 Published: 17 December 2020

Citation:

Rod KA, Patel KF, Kumar S, Cantando E, Leng W, Kukkadapu RK, Qafoku O, Bowden M, Kaplan DI and Kemner KM (2020) Dispersible Colloid Facilitated Release of Organic Carbon

From Two Contrasting Riparian

Sediments. Front. Water 2:560707.

doi: 10.3389/frwa.2020.560707
In aqueous systems, including groundwater, nano-colloids (1-100 nm diameter) and small colloids ( $<450 \mathrm{~nm}$ diameter) provide a vast store of surfaces to which organic carbon $(\mathrm{OC})$ can sorb, precluding its normal bioavailability. Because nanomaterials are ubiquitous and abundant throughout Earth systems, it is reasonable that they would play a significant role in biogeochemical cycles. As such, mineral nano-colloids (MNC) and small colloids, formed through mineral weathering and precipitation processes, are both an unaccounted-for reservoir and unquantified vector for transport of $\mathrm{OC}$ and nutrients and contaminants within watersheds. Water extractions and leaching experiments were conducted under (1) aerobic (ambient) and (2) anaerobic (environmental chamber) conditions for each of two contrasting riparian sediments from (1) Columbia River, Washington and (2) Tims Branch, South Carolina. Water dispersible colloid-adsorbed OC was as high as 48\% of OC for Tims Branch anaerobic batch water extraction and as low as $0 \%$ for Columbia River aerobic batch water extractions. Anaerobic leaching from column experiments yielded higher colloid and OC release rates. Transmission electron microscopy with electron dispersive spectroscopy mapping revealed organic carbon associated with aggregations of nano-particulate silicate minerals and Mossbauer identified nano-particulate goethite. This exploratory study demonstrates that mineral facilitated release of OC in riparian sediments is both significant and variable between locations.

Keywords: organic-carbon, riparian sediment, nano-colloids, dispersible colloids, Columbia River, Tims Branch

\section{INTRODUCTION}

There has already been a recent revolution in understanding how organic carbon (OC) persists in soils. Previous operational definitions and incomplete understanding perpetuated concepts such as "recalcitrant carbon" as an explanation of soil OC persistence. It is now widely accepted that a significant fraction of OC is actually physically and chemically "protected" and is therefore not bioavailable. Bioavailability is restricted by either physical occlusion or by chemical adsorption to mineral surfaces (Schmidt et al., 2011). We are on the frontier of a similar revolution in aqueous systems where the operational definition 
of dissolved OC (DOC; solutes passed through a $0.45 \mu \mathrm{m}$ filter) is incomplete and perhaps incorrect (Yan et al., 2018; Afsar et al., 2020). In aqueous systems, including groundwater, nanocolloids provide a vast store of surfaces to which OC can sorb, precluding its normal bioavailability. Because nanomaterials are ubiquitous and abundant throughout Earth systems (Hochella et al., 2019), it is reasonable that they would play a significant role in biogeochemical cycles. As such, mineral nano-colloids (MNC), formed through mineral weathering and precipitation processes, are both an unaccounted for reservoir and unquantified flux of $\mathrm{OC}$ and nutrients within watersheds. They may also serve as a bridge between organic and inorganic nutrient and contaminant cycles. In groundwater systems OC can adsorb to MNC surfaces (MNC-OC) providing protections from mineralization.

MNCs, defined as between 1 and $100 \mathrm{~nm}$ in at least one dimension, are ubiquitous in the environment and have been found in iron-oxide surface coatings on soil minerals, as well as moving freely in soil and sediment waters (Hochella et al., 2008, 2012; Henderson et al., 2012). Common natural MNCs have been identified as iron-oxides and many other mineral and amorphous families, especially silicates. They have the same chemistry but often subtly different atomic structure than their larger mineral counterparts. Mineral nano-colloids have highly reactive surfaces that sorb $\mathrm{OC}$ to protect it from oxidation and facilitate ion exchange. The colloid morphology and specific sorbed species influence the surface structure and charge of the mineral and, as a result, influence the strength with which OC will bind to the MNC and the transport of MNC-OC through ecosystems. MNCs can be readily transportable between aquatic and terrestrial systems and through soils and sediments.

Mineral nano-colloids may originate from iron oxide nanocrystalline and amorphous iron oxide layers on sediment, which are also known OC adsorption sites (Kleber et al., 2007); iron oxides can release nano-minerals during changes in redox conditions (Henderson et al., 2012). Changing hydrodynamic conditions, particularly fluctuations between extreme wet and dry conditions, accelerate mineral weathering and mineral nanocolloid release. These hydrodynamic fluctuations also solubilize iron surface coatings. However, the interaction between the iron oxides, nano-colloids, and OC has not been fully examined. It has already been demonstrated in soils that chemical adsorption of OC to minerals such as iron oxides provides protection against microbial degradation (Kleber et al., 2007; Schmidt et al., 2011). The surfaces of natural MNCs and colloids can also provide protection in the same manner. This study addresses a gap in watershed science and pore water process understanding by elucidating the dynamic and interconnected role of mineral nano-colloids on OC cycling using highly resolved laboratory experiments.

The objective of this exploratory research was to improve our understanding of the role of water dispersible mineral nanoparticles (MNC) and mineral colloids in biogeochemical cycling and OC transport through watersheds by quantifying release of MNC-OC from riparian sediments from contrasting watersheds. We tested the hypothesis that short-term hydrologic perturbations will release mineral nano-colloids and associated organic carbon to pore water from iron oxide coated sediments.
We conducted batch water extractions and column leaching experiments, under aerobic and anaerobic conditions, to study the release of natural MNCs from sediments, and to quantify the amount of OC associated with colloid fractions and to quantify OC release rates.

\section{METHODS}

\section{Materials}

Riparian capillary fringe sediments were sampled from the Columbia River watershed, near Richland Washington, and Savannah River watershed, near Aiken South Carolina. These rivers represent contrasting systems where Savannah River soils, regionally characterized by Ultisols, are more weathered compared with the Columbia River soil, regionally characterized by Aridisols. The Savannah River watershed was sampled on the banks of Tims Branch tributary, $33^{\circ} 20^{\prime} 15^{\prime \prime} \mathrm{N} 81^{\circ} 43^{\prime} 6^{\prime \prime} \mathrm{W}$, in a sandy $\mathrm{Cg}$ horizon at a depth below $20 \mathrm{~cm}$. The $\mathrm{Cg}$ horizon had distinct redoximorphic features along root channels and was inundated with water during the time of sampling. The bank along the Columbia River was sampled, $46^{\circ} 22^{\prime} 23^{\prime \prime} \mathrm{N} 119^{\circ} 16^{\prime} 19^{\prime \prime}$ $\mathrm{W}$, in a $\mathrm{C}$ horizon below $20 \mathrm{~cm}$. This sandy $\mathrm{C}$ horizon was partially inundated at sampling but had no signs of gleying nor redoximorphic features. However, due to large intermittent releases of water from an upstream hydro dam, this sediment is inundated daily.

Sediment samples were spread out to dry to near field capacity water content so they could be worked with as sediment rather than slurries. Sediments were homogenized before subsampling and characterized for carbon content. Total carbon (TC) was determined by combustion using a VarioMAX Cube (Elementar Analysensysteme $\mathrm{GmbH}$ ). Total organic carbon (TOC) was determined indirectly by first ashing the samples in a muffle furnace $\left(450^{\circ} \mathrm{C}\right.$ for $\left.12 \mathrm{~h}\right)$ to remove $\mathrm{OC}$ and then quantifying total inorganic carbon (TIC) on the VarioMAX Cube (detection limit of $0.1 \mathrm{mg} \mathrm{C}$; measured $\sim 4.3 \mathrm{~g}$ sample). TOC was calculated as the difference between TC and TIC. Total OC for Columbia River sediments was measured at $3.04 \mathrm{~g} \mathrm{~kg}^{-1}$, with TIC at $0.09 \mathrm{~g} \mathrm{~kg}^{-1}$ and for Tims Branch it was $2.11 \mathrm{~g} \mathrm{~kg}^{-1}$, with TIC of $0.06 \mathrm{~g} \mathrm{~kg}^{-1}$.

Synthetic water was fabricated for water extractions and column leaching reactions following established groundwater formulations for Tims Branch and the Hanford site 300 area at the Columbia River. These waters represent contrasting conditions-Tims Branch ground water is relatively dilute with a pH of 5.0 (Strom and Kaback, 1992) compared to Columbia River groundwater, which is saturated relative to calcite and has a pH of 8.05 (Um et al., 2010). Groundwater formulations were used with corresponding sediment (Supplementary Table 1).

\section{Batch Water Extraction}

Surface-adsorbed MNC-OC was extracted from the sediment samples to investigate dispersible colloid and OC composition. The extractions were conducted using $250 \mathrm{~g}$ sediment in $2 \mathrm{~L}$ synthetic groundwater, with gentle agitation at $10 \mathrm{rpm}$ for 7 days (Guigue et al., 2014). Half of the samples were extracted under aerobic (ambient) conditions with container lids (4 L Nalgene) not sealed tight such that gas exchange was possible. The other 
half were treated the same but under anaerobic $\left[\mathrm{N}_{2}(\mathrm{~g})\right]$ conditions after $48 \mathrm{~h}$ equilibration in an anaerobic chamber (Coy Laboratory Products, Grass Lake, MI) with a CAM-12 monitor.

At the end of the extraction, the samples were fractionated for size by centrifugation (Yan et al., 2017) and characterized for colloid concentration and OC concentration. Fractionating in this manner provides detail as to what size fraction has the greatest influence over fate and transport of OC. Centrifugation of water samples was conducted sequentially for the fractions based on silicate densities for the Stokes law calculations (Yan et al., 2017). The fractions included: $<1,000 \mathrm{~nm}$ (160 RCF for $10 \mathrm{~min}$ ); $<450 \mathrm{~nm}$ (555 RCF for $15 \mathrm{~min}$ ); and $<100 \mathrm{~nm}$ (2100 RCF for $80 \mathrm{~min}$ ). This represents the fractions of colloids, common colloid filtration size, and nano-colloid sizes, respectively. A Beckman L8-70M Ultracentrifuge (110,000 RCF for $3 \mathrm{~h}$ ) was used to separate all iron oxides and silicates over $6 \mathrm{~nm}$ from solution (Henneberry et al., 2012). For the purposes of this study we did not differentiate OC associated with MNC and "dissolved" OC in solution for the $<6 \mathrm{~nm}$ fraction. For this research we did not differentiate dissolved OC from colloidal OC independent of minerals that stays in suspension after the centrifugation steps.

Aliquots of fractionated samples were acidified with $1 \%$ nitric acid before analysis for colloid concentration and OC concentration, for the purpose of ensuring inorganic carbon from the high carbonate Hanford water was released and to preserve the samples for future analyses. Colloid concentration was determined by absorption spectra of water samples with a UVVis spectrophotometer (Synergy ${ }^{\mathrm{TM}} \mathrm{Neo} 2 \mathrm{Multi}-$ Mode Microplate Reader, BioTek $\left.{ }^{\circledR}\right)$ with 96 well glass bottom plate (Krystal ${ }^{\mathrm{TM}}$ ). A calibration curve was generated using a suspension of illite at measured concentrations of $3.48,34.8,174$, and $348 \mathrm{mg}$ $\mathrm{L}^{-1}\left(r^{2} \quad 0.99\right)$, and the absorbance was measured at $350 \mathrm{~nm}$ wavelength (Cherrey et al., 2003). Absorbance measurement of the sample and calibration solutions then allowed for estimation of concentration of particles in water samples by applying Beer's law, which assumes spectral adsorption changes proportionally with concentration. Dissolved organic carbon concentration was determined by combustion catalytic oxidation on a Shimadzu TOC-L Analyzer.

Aliquots of $<1,000 \mathrm{~nm}$ fraction were characterized for mineral properties as explained in the next two "Characterization" subsections below.

\section{Aerobic Respiration of Batch Water Extraction}

For aerobic respiration rate quantification, batch water extractions from each condition (aerobic and anaerobic) were collected and centrifuged for $<1,000 \mathrm{~nm}$ colloid fraction. These water samples were immediately transferred and opened in a biosafety cabinet, vortexed, and subsequently allowed to equilibrate with atmospheric air for $2 \mathrm{~h}$ at $21^{\circ} \mathrm{C}$ using a gentle stirrer. Then $43 \mathrm{~mL}$ from each water extract was sterilized by autoclaving at $121^{\circ} \mathrm{C}$ for $20 \mathrm{~min}$, which served as heat-killed controls. Once the autoclaved sediment-suspensions were cooled to room temperature, $40 \mathrm{~mL}$ of each heat-killed control water and two sets of $40 \mathrm{~mL}$ samples (for each extraction condition) were added to $41.5 \mathrm{~mL}$ borosilicate glass bioreactors having pre-attached planar oxygen-sensitive spots $(5 \mathrm{~mm}$ sensor spots; PreSens GmbH, Regensburg, Germany). To prevent any air contamination in the bioreactors, the caps were sealed with a thin layer of parafilm. Dissolved oxygen concentration (mg $\mathrm{L}^{-1}$ ) was monitored in these airtight sensor spot bioreactors for 6 days using an oxygen optical meter assembly (Fibox 3; fiber optic oxygen transmitter, a polymer optical fiber) and recorded using PST3v602 software (PreSens Precision Sensing $\mathrm{GmbH}$, Regensburg, Germany). Briefly, capped bioreactors were vortexed for $20 \mathrm{~s}$ and three readings of dissolved oxygen $(\mathrm{mg}$ $\mathrm{L}^{-1}$ ) were recorded at the interval of $5 \mathrm{sec}$. After recording the dissolved oxygen, the bioreactors were immediately transferred to a shaker (300 rpm; platform shaker; Innova ${ }^{\circledR} 2100$; New Brunswick Scientific) in the environmental chamber with controlled temp $\left(21^{\circ} \mathrm{C}\right)$ in dark. Respiration rates were calculated as the slope of the linear regression between dissolved oxygen concentration and incubation time for each bioreactor. The linear regression generates a negative slope which is a function of the oxygen uptake rate and therefore aerobic respiration rates $\left(\mathrm{mg} \mathrm{O}_{2} \mathrm{~L}^{-1} \mathrm{~h}^{-1}\right)$. A two-point manual calibration was performed prior to the sample analysis per PreSens guidelines using oxygen-free and oxygen-saturated water to ensure the quality control check.

\section{Characterization of Batch Water Extracts: TEM-EDS (Colloids), Zeta Potential}

Transmission electron microscopy with electron dispersive spectroscopy (TEM-EDS) was used for visual and elemental identification of MNCs (Wheatland et al., 2017). The particles from water samples were collected onto a 300 mesh copper TEM grid with lacey carbon support film using Beckman ultracentrifuge (274,400 RCF, 24h). A JEOL 2100 TEM/Scanning-TEM operating at $200 \mathrm{kV}$, coupled with EDS and selected-area electron diffraction (SAED), were applied to characterize the morphology, composition, and crystal structures of the particles.

Since surface electric potential is significant for the adsorption and desorption of MNC to sediment, we measured the colloid suspensions zeta potentials (Appel et al., 2003). Samples were sonicated for $10 \mathrm{~min}$ to disperse them, then analyzed for zeta potential using a Malvern Zetasizer Nano ZS.

\section{Colloid Characterization From Tims Branch Sediment: Mössbauer, $\mu-X R D$, and SEM-EDX}

For the Tims Branch sediment aerobic extraction with groundwater, the colloid concentration was high enough to do additional mineral characterization using $\mu$-XRD, SEM-EDX, and Mössbauer spectroscopy. These analyses were performed on the $<1,000 \mathrm{~nm}$ fraction, collected by spinning samples in an ultra-centrifuge to remove colloids from suspension. The solution was then decanted and the remaining water was allowed to evaporate in a fume hood. 
Powder diffraction was performed using a Panalytical X'Pert Bragg-Brentano diffractometer, $\mu$-XRD, with $\mathrm{Cu}-\mathrm{K} \alpha$ radiation $(\lambda$ $=1.5418 \AA$ ), a graphite post-diffraction monochromator, and variable divergence and anti-scatter slits (illuminated length = $10 \mathrm{~mm}$ ). Minerals were identified using JADE software (Materials Data Incorporated, CA) by comparison with reference patterns in the database maintained by the International Center for Diffraction Data (PA).

Mössbauer measurements were carried out on the colloid sample using a SEE Co Mössbauer setup (Edina, MN), a Ritverc ${ }^{57} \mathrm{Co} / \mathrm{Rh}$ gamma energy source (75-mCi, initial strength; St. Petersburg, Russia), a scintillation NaI(T1) SGS-2 detector (Ritverc), a closed-cycle cryostat SHI-850 (Janis Research Co., Inc., Wilmington, MA), and a Sumitomo CKW-21 He compressor unit (Allentown, PA). The transmitted counts were stored in a multichannel scalar (MCS) as a function of the energy (transducer velocity) using a 1024-channel analyzer. Calibration spectra were obtained with a $7-\mu \mathrm{m}$-thick Fe foil placed in the same position as the samples to minimize any geometry errors. The raw data were folded to 512 channels to provide a flat background and a zero-velocity position corresponding to the center shift (CS) of a metal Fe foil at room temperature (RT). The Mössbauer spectroscopy data were modeled with Recoil software (University of Ottawa, Canada) using a Voigtbased structural fitting routine (Rancourt and Ping, 1991). The sample preparation (type of the sample holder, etc.) was similar to the procedure reported in Peretyazhko et al. (2012), except the samples were dispersed in powdered sugar instead of petroleum jelly.

Scanning Electron Microscopy (SEM) analyses were conducted with a FEI Helios NanoLab 600i field emission electron microscope. A small amount of dried colloid powder was mounted on a SEM aluminum stub and were coated with a $10 \mathrm{~nm}$ carbon layer by thermal evaporation using a $108 \mathrm{C}$ Auto Carbon Coater (Ted Pella, Inc.). Particle morphology was examined using a secondary electron Everhart-Thornley detector

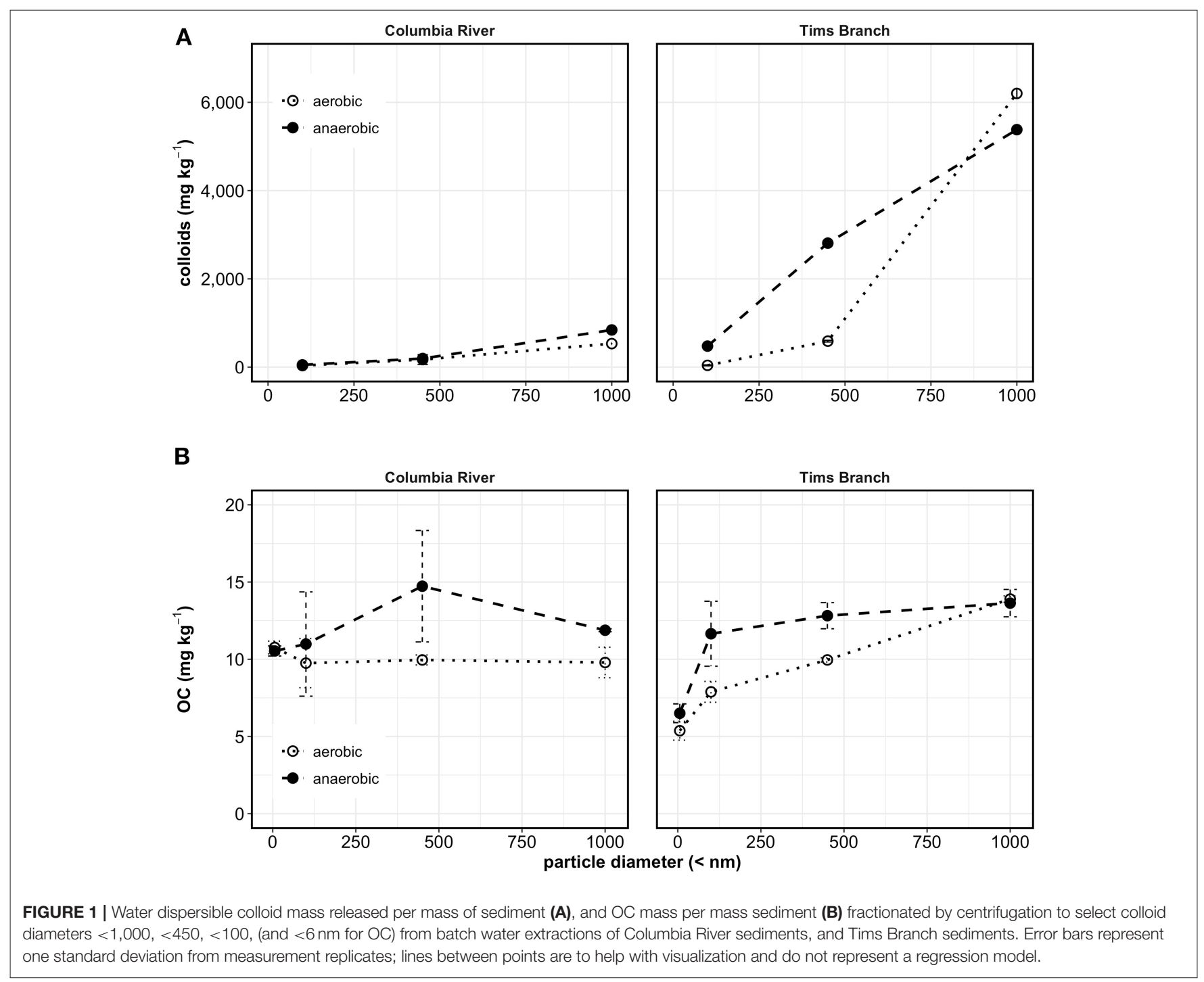




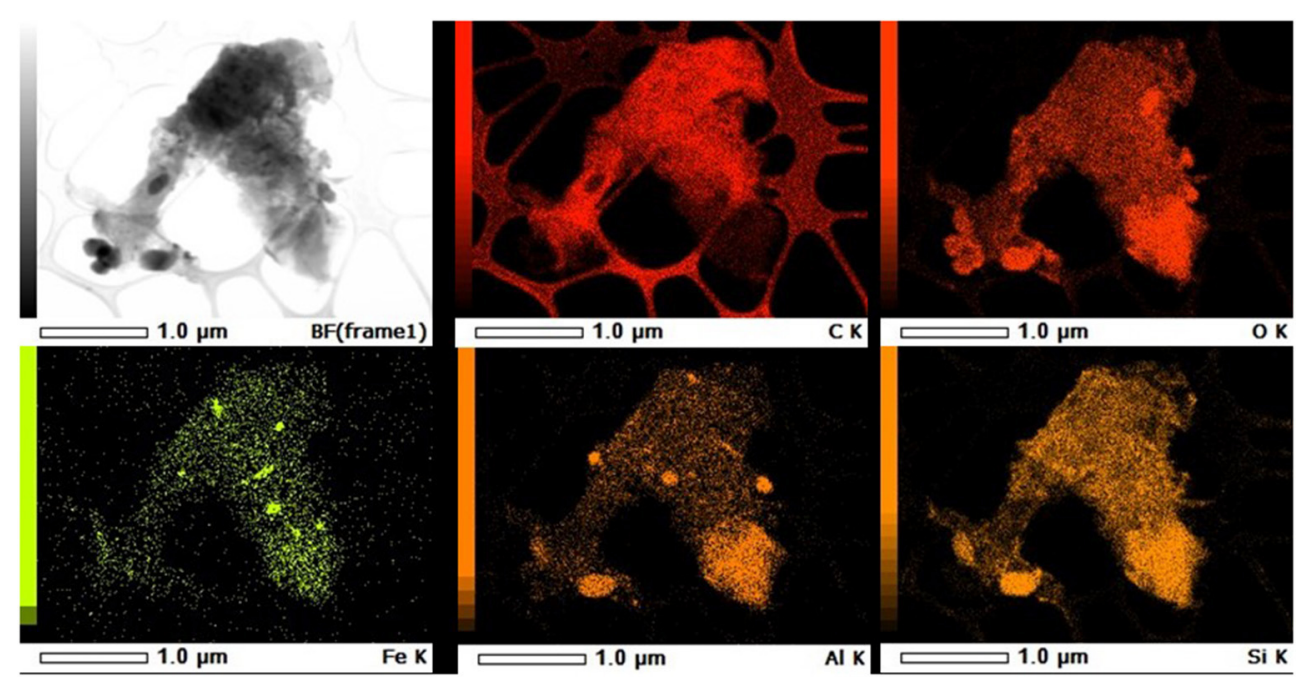

FIGURE 2 | Representative TEM-EDS map of water extracted colloids from Tims Branch Sediment Anaerobic sample. First image is TEM. The remaining images are EDS elemental maps of the same image showing relative intensity of each element. Scale and element are listed in footer for each image.

(ETD) in a field free mode at an acceleration voltage of $3-5 \mathrm{kV}$ and a current of $0.086-0.17 \mathrm{nA}$ at $4 \mathrm{~mm}$ working distance. Higher magnification images were collected using through-lens-detector (TLD) with same voltage/current as described above. Energy dispersive $\mathrm{X}$-ray (EDX) spectra were collected with X-Max 80 $\mathrm{mm}^{2}$ Silicon Drift Detector (SDD) from Oxford Instruments. The EDX analyses were performed at $10-20 \mathrm{kV}$ voltage and 2-3 nA current. Oxford INCA software was used to perform point spectrum analyses and create compositional maps.

\section{Column Leaching}

Leaching tests were conducted to measure relative release rates of OC and colloids from the sediments. Columns were packed with sediment into $75 \mathrm{~mL}$ internal volume glass columns with $2.54 \mathrm{~cm}$ inner diameter. Two columns were packed for each sediment type, to a resultant porosity of 0.48 for the Columbia River columns and 0.44 for the Tims Branch columns. One from each column pair was run under anaerobic conditions, the other aerobic, as described in "Batch water extraction" section. Syringe pumps (IMI-Kloehn) were used to deliver synthetic groundwater at a rate of three pore volumes per day. Effluent was collected twice a day and centrifuged for fraction $<450 \mathrm{~nm}$ silicate as detailed above. The effluent was analyzed for colloid concentration by UV-Vis and the OC content measured by a Shimadzu TOC-L Analyzer.

\section{RESULTS AND DISCUSSION}

\section{Fractionation of Released Colloids From Batch Extractions}

Release of colloids from Columbia River sediments (Figure 1A) was $58 \%$ greater for anaerobic than aerobic extracts for the $<1,000 \mathrm{~nm}$ fraction and $45 \%$ greater for the $<100 \mathrm{~nm}$ fraction. The higher release during the induced anoxia was expected as it has been well-documented in the literature and is attributed to the reductive dissolution of cementing ferric iron oxides to more soluble ferrous iron, resulting in the release of aggregated colloids (Henderson et al., 2012; Liang et al., 2019). Tims Branch sediments released an order of magnitude more colloids due to anoxia for the $<100 \mathrm{~nm}$ fraction but the $<1,000 \mathrm{~nm}$ fraction had $15 \%$ more released from aerobic extraction compared to anaerobic (Figure 1A). The lower value for the anaerobic extraction may be attributed to aggregation and settling before analysis or subsample variability. Aggregation was noticed at multiple points of analysis, and occurred despite additional preparation (acidification, sonication) before analysis. The release of colloids from Tims Branch sediments was three to ten times greater than from Columbia River sediments in all instances except for $<100 \mathrm{~nm}$ aerobic extraction which was $14 \%$ higher for Tims Branch. Higher colloid concentrations are most likely due to a combination of the very low ionic strength of the SRS artificial groundwater, $0.2 \mathrm{mM}$ (compared to $6.4 \mathrm{mM}$ Hanford artificial groundwater), and the high concentrations of highly dispersible OC-coated Feoxyhydroxides and fine kaolinite particles common in these weathered soils (Kaplan et al., 1993, 1997).

\section{Colloid Mineralogy}

Colloids collected from each of the extractions were imaged using TEM-EDX (Figure 2 and Supplementary Figures 1-4). All of the images displayed aggregations of minerals that had primarily round scale-like morphology with the occasional elongated mineral (Supplementary Figures 2, 4). The minerals had high relative element concentrations of $\mathrm{Si}, \mathrm{Al}$, and $\mathrm{O}$, suggesting that these are primarily silicate minerals (Figure 2 and Supplementary Figures 1-4). Tims Branch soils have documented kaolinite as a significant colloid (Kaplan et al., 1993, 1997) and clays from the Hanford 300 area have been reported as dominantly muscovite, smectite, and clinochlore (Um et al., 2010). The EDX showed even distribution of Fe 
across most of the minerals for the Columbia River colloids (Supplementary Figures 1, 2), whereas the Tims Branch colloids had small concentrated points scattered over the mineral surfaces (Figure 2 and Supplementary Figures 3, 4). It is possible that these points with high $\mathrm{Fe}$ concentration are nano colloids aggregated with the larger minerals. The presence of $\mathrm{C}$ on the Tims Branch colloids (Figure 2 and Supplementary Figures 3, 4) was much more evident compared to the Columbia River colloids (Supplementary Figures 1, 2). Organic coatings on colloids from soils of the same region as Tims Branch have previously been noted (Kaplan et al., 1997).

The $\mu$-XRD pattern of colloids from Tims Branch aerobic water extraction (Supplementary Figure 5) was crystalline with sharp peaks for kaolinite. There were also peaks for quartz and gibbsite, minor illite or montmorillonite, and trace hematite. The presence of these minerals is consistent with previous work on soils from this watershed (Kaplan et al., 1993, 1997). The morphology of the minerals from SEM analysis showed that most of the material was rounded flakes and the EDX data shows high relative concentrations of $\mathrm{Si}, \mathrm{Al}$, and $\mathrm{O}$ as would be expected in a silicate clay mineral like kaolinite (Supplementary Figure 6). High relative Fe concentrations point to Fe- "coatings" adsorbed on the larger minerals.

The Mössbauer spectra from Tims Branch aerobic water extraction (Supplementary Figure 7) confirms the presence of hematite, with $<3 \%$ Fe being hematite. Approximately $80 \%$ of the sample's Fe was nanoparticulate goethite $(<10 \mathrm{~nm})$ with a somewhat complex particle-size distribution, but appeared to be a mixture of three different pools of varying particle size/carbon coating content (Bhattacharyya et al., 2018; Arredondo et al., 2019). More importantly, the sample was free of poorly crystalline/amorphous Fe-oxide minerals, such as ferrihydrite. The remaining $\mathrm{Fe}$ in the colloids sample $(\sim 20 \%)$ was likely associated with silicate minerals-primarily (14.9\%) as $\mathrm{Fe}(\mathrm{III})$, but there was some (4.8\%) $\mathrm{Fe}(\mathrm{II})$.

The silicates, kaolinite, and quartz identified from Tims Branch should have lower adsorptive capacity compared to the previously identified muscovite, smectite, and clinochlore at Columbia River (Um et al., 2010). This suggests a significant role that the Fe-particles and $\mathrm{Fe}$-coatings on silicates have on the adsorption of the OC.

\section{Fractionation of Released Mineral Colloid-Associated OC From Batch Extractions}

Similar to colloids, there was more release of OC from the anaerobic extraction (Figure 1B), suggesting that it was released during induced anoxia by similar desorption mechanisms (Adhikari et al., 2017; Han et al., 2019). Using the $<6 \mathrm{~nm}$ fraction as an estimated cut off for dissolved OC (DOC), the OC associated with Columbia River mineral colloids was $29 \%$ of the maximum measured OC (26\% in $100-450 \mathrm{~nm}$ fraction, $3 \%$ in $6-100 \mathrm{~nm}$ fraction of the maximum measured OC for that extraction) for the anaerobic extraction. For the Columbia River aerobic extracts, the $<6 \mathrm{~nm}$ fraction had $10.8 \mathrm{mg} \mathrm{kg}^{-1}$ DOC, compared to the mineral colloid fraction $<100 \mathrm{~nm}$ of $9.7 \mathrm{mg}$ $\mathrm{kg}^{-1}$, suggesting that there was no mineral colloid-associated $\mathrm{OC}$ in these extracts (Figure 1B). As with the colloids, some aggregation and settling may have impacted the measurement of $\mathrm{OC}$ as can be seen for the $<1,000 \mathrm{~nm}$ fraction from the Columbia River sediments (Figure 1B). Alternately this lower value for $<1,000 \mathrm{~nm}$ was data noise as evidenced by the wide measurement replicate error bars for the $<450 \mathrm{~nm}$ sample (Figure 1B). For Tims Branch, $48 \%$ of anaerobic extracted OC was associated with mineral colloids with $38 \%$ in the $6-100 \mathrm{~nm}$ fraction and $8 \%$ in the $100-450 \mathrm{~nm}$ fraction (Figure 1B). The mineral colloid associated OC released during aerobic water extraction was $40 \%$ of total released, with $18 \%$ in the $6-100 \mathrm{~nm}$ fraction and $15 \%$ in the $100-450 \mathrm{~nm}$ fraction, and $7 \%$ in the $450-1,000 \mathrm{~nm}$ fraction.

Reducing conditions have been known to desorb OC from $\mathrm{Fe}$ colloids (Adhikari et al., 2017; Han et al., 2019), and the different responses for the two sediments may be explained by the natural redox conditions at these sites. The subsurface along the Hanford reach of the Columbia River is largely aerobic, so contribution of mineral colloids and MNCs to OC cycling may be minimal based on the results from this experiment. However, Tims Branch sediments had redoximorphic features from repeated oxic and anoxic periods, which could result in fluctuating release concentrations of MNC-OC and OC associated with small colloids.

Microbial degradation reducing the measured OC was a concern for impacting the measurement of OC concentrations

TABLE 1 | Batch water properties (microbial respiration, zeta potential) and column effluent data (OC and colloid release rates, pore velocity) for Columbia River sediment and Tims Branch sediment reacted with groundwater under aerobic and anaerobic conditions.

\begin{tabular}{|c|c|c|c|c|c|c|c|}
\hline Sample & Condition & $\begin{array}{l}\text { Microbial respiration rate } \\
\qquad\left(\mathrm{mg} \mathrm{L}^{-1} \mathrm{hr}^{-1}\right)\end{array}$ & \multicolumn{2}{|r|}{ Batch } & \multicolumn{2}{|c|}{ Column } & $\begin{array}{l}\text { Calculated pore velocity } \\
\qquad\left(\mathrm{m} \mathrm{d}^{-1}\right)^{\star \star}\end{array}$ \\
\hline \multirow[t]{2}{*}{ Columbia River } & Aerobic & 0.011 & 7.38 & -8.77 & $0.92 ; 0.99$ & $2.8 ; 0.98$ & 0.876 \\
\hline & Anaerobic & 0.009 & 6.95 & -12.8 & $1.36 ; 0.75$ & 8.8; 0.98 & 0.894 \\
\hline Tims Branch & Aerobic & 0.011 & 6.02 & -8.43 & $0.36 ; 0.90$ & $13.4 ; 0.87$ & 0.973 \\
\hline
\end{tabular}

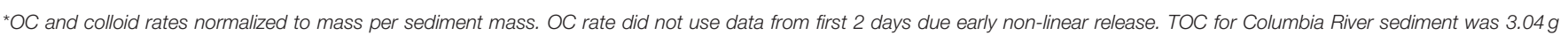

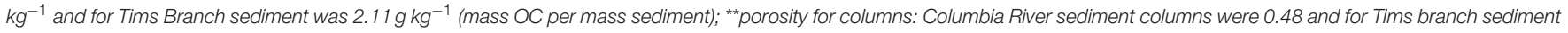
columns were 0.44. Column data plots for colloid and OC release are provided in Supplementary Figure 8. 
and calculated OC release rates. However, respiration was very low in all water samples (Table 1) and there was no effective difference between the conditions in this experiment.

\section{Leaching Rates of Colloids and OC From Columns}

The initial release of colloids for Columbia River sediment columns within the first day was lower than colloids from Tims Branch sediment columns, but the OC had a proportionally larger release from Columbia River columns (Supplementary Figure 8). This initial release difference is likely due to desorption of the OC occurring more rapidly than detachment of colloids.

Following the initial release there was a sustained release, from day 1 to 5, of colloids and OC from the columns that showed a higher release rate for anaerobic conditions compared to aerobic reaction conditions (Table 1). The increase in release rates with anaerobic extraction was expected and coincided with more negative zeta potential for the anaerobic reacted water extractions (Table 1). Li et al. (2013) found that colloids amended with organics decreased the zeta potential and the colloid-OC material transported more readily through sediment. In our study, the Tims Branch colloids had a higher release rate compared to the Columbia River colloids (Table 1), which could be due to a combination of factors. This higher release could be due to a more dilute groundwater, greater repulsive forces due to lower zeta potential for the organic coated colloids (Table 1 and Figure 2), or there was an abundance of colloids which would be present in a region with highly weathered soils. The matrix in both of these sediments is sand with large pore spaces that would allow easy transport of the colloids with minimal surface interaction or straining. Ultimately this demonstrates that, even when there is OC adsorbed to-colloids, release of the bulk OC differs from that of the bulk colloids regardless of whether the release is aerobic or anaerobic.

\section{ENVIRONMENTAL IMPLICATIONS}

Colloids can play a significant role in the fate and transport of natural OC through watersheds, particularly in redox dynamic environments where reductive dissolution of Fe minerals can facilitate increased concentrations of colloids within porewaters. This is particularly relevant for Tims Branch sediments that, upon reduction, can release up to $48 \%$ of their OC in association with colloids. Most of the OC was attached to MNCs, which with their size of $<100 \mathrm{~nm}$ can be transported readily through sediment, making nano-colloid-facilitated transport a significant agent of $\mathrm{C}$ biogeochemical cycling. However, this may not be the case in all riparian sediments such as found from Columbia River sediments under aerobic conditions where there is no measurable adsorption of OC to colloids of any size. The importance of colloidal and MNC facilitated transport of OC in watersheds appears to be location specific. Since MNCs have high surface charge, they can potentially limit bioavailability of OC while adsorbed to them and while being transported through a watershed. This control on the bioavailability of OC while adsorbed to MNCs must be accounted for in modeling
OC transport as the transport of colloids is different than dissolved OC.

\section{DATA AVAILABILITY STATEMENT}

The raw data supporting the conclusions of this article will be made available by the authors, without undue reservation, to any qualified researcher.

\section{AUTHOR CONTRIBUTIONS}

KR: conceptualized, field data collection, experimentation, data analysis, and writing. KP: data generation, data analysis, and writing. SK: respiration experiments and writing. EC: nano-colloid characterization, TEM, and writing. WL: nanocolloid characterization, zeta potential, and writing. RK: mossbauer, analysis, and writing. OQ: SEM, analysis, and writing. MB: UXRD, analysis, and writing. DK: field data collection, senior review, and writing. KK: senior review and writing. All authors contributed to the article and approved the submitted version.

\section{FUNDING}

This work was supported by Pacific Northwest National Laboratory SEED-LDRD [DE-AC05-76RLO 1830, AC0206CH11357, AC09-08SR22470, and NSF(ECCS 1542100)].

\section{ACKNOWLEDGMENTS}

This research described in this paper was conducted under the Laboratory Directed Research and Development Program at Pacific Northwest National Laboratory (PNNL). A portion of this research was performed in the W. R. Wiley Environmental Molecular Sciences Laboratory (EMSL), a national scientific user facility sponsored by the Office of Biological and Environmental Research (BER) and located at PNNL. PNNL is a multiprogram national laboratory operated by Battelle for the U.S. Department of Energy (DOE) under contract DE-AC05-76RLO 1830. Support for DK and KK was provided by the Argonne Wetland Hydrobiogeochemistry Science Focus Area funded by the Subsurface Biogeochemical Research Program of the Office of Biological and Environmental Research, Office of Science, U.S. Department of Energy (DOE), under contracts DEAC02-06CH11357 (Argonne National Laboratory) and AC0908SR22470 (Savannah River Nuclear Solutions). This work was supported by the Virginia Tech National Center for Earth and Environmental Nanotechnology Infrastructure (NanoEarth), a member of the National Nanotechnology Coordinated Infrastructure (NNCI), supported by NSF (ECCS 1542100 and ECCS 2025151).

\section{SUPPLEMENTARY MATERIAL}

The Supplementary Material for this article can be found online at: https://www.frontiersin.org/articles/10.3389/frwa. 2020.560707/full\#supplementary-material 


\section{REFERENCES}

Adhikari, D., Zhao, Q., Das, K., Mejia, J., Huang, R., Wang, X., et al. (2017). Dynamics of ferrihydrite-bound organic carbon during microbial Fe reduction. Geochim. Cosmochim. Acta 212, 221-233. doi: 10.1016/j.gca.2017.06.017

Afsar, M. Z., Goodwin, C., Beebe, T. P., Jaisi, D. P., and Jin, Y. (2020). Quantification and molecular characterization of organo-mineral associations as influenced by redox oscillations. Sci. Total Environ. 704:135454. doi: 10.1016/j.scitotenv.2019.135454

Appel, C., Ma, L. Q., Rhue, R. D., and Kennelley, E. (2003). Point of zero charge determination in soils and minerals via traditional methods and detection of electroacoustic mobility. Geoderma 113, 77-93. doi: 10.1016/S0016-7061(02)00316-6

Arredondo, M. G., Lawrence, C. R., Schulz, M. S., Tfaily, M. M., Kukkadapu, R., Jones, M. E., et al. (2019). Root-driven weathering impacts on mineral-organic associations in deep soils over pedogenic time scales. Geochim. Cosmochim. Acta 263, 68-84. doi: 10.1016/j.gca.2019.07.030

Bhattacharyya, A., Campbell, A. N., Tfaily, M. M., Lin, Y., Kukkadapu, R. K., Silver, W. L., et al. (2018). Redox fluctuations control the coupled cycling of iron and carbon in tropical forest soils. Environ. Sci. Technol. 52, 14129-14139. doi: 10.1021/acs.est.8b03408

Cherrey, K. D., Flury, M., and Harsh, J. B. (2003). Nitrate and colloid transport through coarse Hanford sediments under steady state, variably saturated flow. Water Resour. Res. 39:1165. doi: 10.1029/2002WR0 01944

Guigue, J., Mathieu, O., Lévêque, J., Mounier, S., Laffont, R., Maron, P. A., et al. (2014). A comparison of extraction procedures for water-extractable organic matter in soils. Eur. J. Soil Sci. 65, 520-530. doi: 10.1111/ejss. 12156

Han, L., Sun, K., Keiluweit, M., Yang, Y., Yang, Y., Jin, J., et al. (2019). Mobilization of ferrihydrite-associated organic carbon during $\mathrm{Fe}$ reduction: adsorption versus coprecipitation. Chem. Geol. 503, 61-68. doi: 10.1016/j.chemgeo.2018.10.028

Henderson, R., Kabengi, N., Mantripragada, N., Cabrera, M., Hassan, S., and Thompson, A. (2012). Anoxia-induced release of colloid- and nanoparticlebound phosphorus in grassland soils. Environ. Sci. Technol. 46, 11727-11734. doi: $10.1021 /$ es302395r

Henneberry, Y. K., Kraus, T. E. C., Nico, P. S., and Horwath, W. R. (2012). Structural stability of coprecipitated natural organic matter and ferric iron under reducing conditions. Org. Geochem. 48, 81-89. doi: 10.1016/j.orggeochem.2012.04.005

Hochella, M. F., Aruguete, D., Kim, B., and Madden, A. S. (2012). "Naturally occuing inorganic nanoparticles: general assessment and a global budget for one of earths's last unexpored major geochemical components," in Nature's Nanostructures, eds A. S. Barnard and H. Guo (Victoria: Pan Stanford Publishing Pte. Ltd.), 1-42.

Hochella, M. F., Lower, S. K., Maurice, P. A., Penn, R. L., Sahai, N., Sparks, D. L., et al. (2008). Nanominerals, mineral nanoparticles, and Earth systems. Science 319, 1631-1635. doi: 10.1126/science.1141134

Hochella, M. F., Mogk, D. W., Ranville, J., Allen, I. C., Luther, G. W., Marr, L. C., et al. (2019). Natural, incidental, and engineered nanomaterials and their impacts on the Earth system. Science 363:eaau8299. doi: 10.1126/science.aau8299

Kaplan, D. I., Bertsch, P. M., and Adriano, D. C. (1997). Mineralogical and physicochemical differences between mobile and nonmobile colloidal phases in reconstructed pedons. Soil Sci. Soc. Am. J. 61, 641-649. doi: 10.2136/sssaj1997.03615995006100020038x
Kaplan, D. I., Bertsch, P. M., Adriano, D. C., and Miller, W. P. (1993). Soil-borne mobile colloids as influenced by water flow and organic carbon. Environ. Sci. Technol. 27:6. doi: 10.1021/es00043a021

Kleber, M., Sollins, P., and Sutton, R. (2007). A conceptual model of organo-mineral interactions in soils: self-assembly of organic molecular fragments into zonal structures on mineral surfaces. Biogeochemistry 85, 9-24. doi: 10.1007/s10533-007-9103-5

Li, D., Chang, H. S., Seaman, J. C., and Kaplan, D. I. (2013). Effects of matrix heterogeneity and aqueous humic acid on transport and deposition of mineral colloids in sandy sediments. J. Environ. Chem. Eng. 1, 875-883. doi: 10.1016/j.jece.2013.07.032

Liang, X. L., Radosevich, M., Loffler, F., Schaeffer, S. M., and Zhuang, J. (2019). Impact of microbial iron oxide reduction on the transport of diffusible tracers and non-diffusible nanoparticles in soils. Chemosphere 220, 391-402. doi: 10.1016/j.chemosphere.2018.12.165

Peretyazhko, T. S., Zachara, J. M., Kukkadapu, R. K., Heald, S. M., Kutnyakov, I. V., Resch, C. T., et al. (2012). Pertechnetate (TcO4-) reduction by reactive ferrous iron forms in naturally anoxic, redox transition zone sediments from the Hanford Site, USA. Geochim. Cosmochim. Acta. 92, 48-66. doi: 10.1016/j.gca.2012.05.041

Rancourt, D. G., and Ping, J. Y. (1991). Voigt-based methods for arbitrary-shape static hyperfine parameter distributions in mossbauer-spectroscopy. Nuclear Instrum. Methods Phys. Res. Sect. B Beam Interact. Mater. Atoms 58, 85-97. doi: 10.1016/0168-583X(91)95681-3

Schmidt, M. W. I., Torn, M. S., Abiven, S., Dittmar, T., Guggenberger, G., Janssens, I. A., et al. (2011). Persistence of soil organic matter as an ecosystem property. Nature 478, 49-56. doi: 10.1038/nature10386

Strom, R., and Kaback, D. (1992). SRP Baseline Hydrogeologic Investigation: Aquifer Characterization Groundwater Geochemistry of the Savannah River Site and Vicinity, WSRC-RP-92-450. Aiken, SC: Westinghouse Savannah River Company. doi: 10.2172/10103229

Um, W., Zachara, J. M., Liu, C. X., Moore, D. A., and Rod, K. A. (2010). Resupply mechanism to a contaminated aquifer: a laboratory study of U(VI) desorption from capillary fringe sediments. Geochim. Cosmochim. Acta 74, 5155-5170. doi: 10.1016/j.gca.2010.02.001

Wheatland, J. A. T., Bushby, A. J., and Spencer, K. L. (2017). Quantifying the Structure and composition of flocculated suspended particulate matter using focused ion beam nanotomography. Environ. Sci. Technol. 51, 8917-8925. doi: 10.1021/acs.est.7b00770

Yan, J., Manelski, R., Vasilas, B., and Jin, Y. (2018). Mobile colloidal organic carbon: an underestimated carbon pool in global carbon cycles? Front. Environ. Sci. 6:148. doi: 10.3389/fenvs.2018.00148

Yan, J., Meng, X., and Jin, Y. (2017). Size-dependent turbidimetric quantification of suspended soil colloids. Vadose Zone J. 16, 1-8. doi: 10.2136/vzj2016.10.0098

Conflict of Interest: The authors declare that the research was conducted in the absence of any commercial or financial relationships that could be construed as a potential conflict of interest.

Copyright (c) 2020 Rod, Patel, Kumar, Cantando, Leng, Kukkadapu, Qafoku, Bowden, Kaplan and Kemner. This is an open-access article distributed under the terms of the Creative Commons Attribution License (CC BY). The use, distribution or reproduction in other forums is permitted, provided the original author(s) and the copyright owner(s) are credited and that the original publication in this journal is cited, in accordance with accepted academic practice. No use, distribution or reproduction is permitted which does not comply with these terms. 